\title{
Non-contact Method for Analysis of Cavitating Flows
}

\author{
Ignacijo Biluša , Benjamin Bizjan*b, Luka Lešnik ${ }^{a}$, Brane Širok ${ }^{b}$, Boštjan Pečnikc, Matevž Dular ${ }^{b}$ \\ a University of Maribor, Faculty of Mechanical engineering, Smetanova ulica 17, 2000 Maribor, Slovenia \\ ${ }^{b}$ University of Ljubljana, Faculty of Mechanical engineering, Aškerčeva 6, 1000 Ljubljana, Slovenia \\ c Gorenje d.d., Partizanska 12, 3503 Velenje, Slovenia \\ *Corresponding author. Email address: benjamin.bizjan@fs.uni.lj.si; Phone: +386 14771423
}

\begin{abstract}
This paper presents a novel non-contact method for simultaneous analysis of pressure and velocity conditions in cavitating flows. The method (implemented in our software ADMflow) is based on high-speed camera flow visualization and was evaluated in an experiment with ultrasonically induced acoustic cavitation of different intensities. Attached cavitation with clearly visible cavitation structures occurred on the tip of an ultrasonic probe immersed in distilled water. Using the high-speed imaging data, pressure fluctuations were calculated by a computer-aided algorithm based on the Brennen's theory of cavitation cloud kinematics and a modified version of the Rayleigh-Plesset equation. Reference measurements of pressure pulsations were conducted by a hydrophone installed at the bottom of the liquid container. The analysis of cavitation structure dynamics was complemented by calculation of velocity fields from the imaging data, the algorithm for which is based on the advectiondiffusion equation. Calculated pressure fluctuations were analyzed in the spatial, temporal and spectral domain. Presented results indicate a strong correlation between the fields of velocity and pressure fluctuations during the growth and collapse of cavitation structures. A comparison of time series and power spectra demonstrates that our cavitation analysis method is in a reasonably good agreement with results of the reference measurement methods and can therefore be used for non-contact analysis of pressure and velocity conditions in cavitating flows.
\end{abstract}

Keywords: Acoustic cavitation, sonotrode, pressure measurement, hydrophone, computeraided visualization, image velocimetry

\section{Introduction}

Cavitation is a highly complex flow phenomenon in which various vapor structures are formed at low-pressure regions of the liquid, then collapsing violently, causing locally very high values of pressure and velocity, and often resulting in erosion on exposed surfaces [1]. Cavitation can be induced hydrodynamically (i.e. due to the kinetic energy of the flow) or ultrasonically (e.g. on an oscillating ultrasonic probe). Accurate measurements of flow variables such as pressure and velocity are crucial in experimental studies, which remain important in research of complex cavitation phenomena despite the rapid development of numerical cavitation modeling methods. However, due to the harsh operating conditions and the multiphase flow nature, the selection of flow analysis methods is limited. The methods most commonly used for this purpose include local measurements of pressure (e.g. by hydrophones) and temperature, as well as optical (flow visualization-based) which are becoming increasingly popular with the rapid development of high-speed imaging 
technologies (Verhaagen, Rivas [2]). The main advantage of flow visualization techniques is the ability to obtain a large amount of flow dynamics data with a single measurement. However, the main challenge remains in post-processing of the high-speed imaging data, as there is still no known method capable of directly calculating the pressure fields within the liquid. As a response to this issue, this paper presents and evaluates a novel computer-aided algorithm for simultaneous calculation of velocity and pressure fields in cavitating flows.

With that said, numerous flow visualization studies were so far performed on cavitating hydrofoils (Wade and Acosta [3], Kubota et al. [4], Reisman and Brennen [5], Franc et al. [6], Kato et al. [7]) and for ultrasonic cavitation (Tzanakis et al. [8], Leppinen and Dalziel [9]), with the aim to improve the optical measurement methods and the understanding of observed cavitation phenomena. Reisman and Brennen [5], apart from the flow visualization, also performed a spectral analysis of hydrophone-measured pressure fluctuations, and classified them to a local and global scale. Reisman et al. [10] applied high-speed flow visualization to study the highly complex mechanism of shock wave formation in cavitation clouds, and related mechanisms of noise formation and erosion. Osterman et al. [11] conducted simultaneous pressure measurements and visualization of incipient cavitation in a valve, demonstrating the visualization method to be more sensitive to cavitation and its signal independent of the operating pressure. An experiment with similar measurement techniques has recently been performed by Tzanakis et al. [8] to study ultrasonically induced acoustic cavitation in different liquids. A different approach was taken by Kato et al. [7], who combined the visualization of a cavitating flow with PIV velocity field measurements. Apart from pressure and velocity, another important property of cavitating flows is the volume fraction of the vapor phase. For typical high-speed imaging setups lit from the side opposite to the camera, light attenuation methods are used most commonly (Leppinen and Dalziel [9]). Despite the fact that hydrodynamic and the ultrasonic cavitation have a different cause, the principles which govern the hydrodynamic bubble and acoustic bubble are basically the same (Franc \& Michel [12]).

For the most accurate characterization of cavitating phenomena, it is desired to combine measurements of different flow properties in the same experiment. However, using a different measurement method and device for each flow property (e.g. hydrophones for pressure, PIV for velocity and high-speed cameras for visualization) adds to the complexity and price of the measurement set-up, and is often impractical. The rapid development of high-speed imaging devices and flow analysis algorithms in the recent years has created the opportunity to measure several cavitating flow properties from a single image sequence, and possibly replace some of the more conventional measurement techniques. Speaking of the flow velocity measurement, it is known that the PIV and similar correlation-based methods require a quite complex measurement setup and may not always perform well when used with normal imaging sequences. The flow velocimetry methods based on the optical flow theory present a more robust alternative (i.e. less sensitive to the quality of imaging setup). An extensive overview of the optical flow methods is presented in works of Barron et al. [13] and Zimmer et al. [14]. Recently, a flow velocimetry method based on the optical flow and the advection-diffusion equation was developed by our research group at the University of Ljubljana (Bajcar et al. [15], Bizjan et al. [16][17]), and validated on several laboratory and industrial flow phenomena. This method will also be presented and applied in this manuscript.

Unlike velocity measurements, little research has been dedicated to the calculation of fluid pressure from flow visualization images in cavitating flows. Nevertheless, the mathematical 
modeling of bubble and cloud cavitation is extensively covered in the literature. The basic mathematical formulation for dynamics of cavitating spherical bubbles is the RayleighPlesset equation (Plesset and Prosperetti [18]). The equation was later applied to the models for cloud cavitation (Brennen [1], d'Agostino and Brennen [19], Wang and Brennen [20], Shimada and Kobayashi [21]), which is much more common in practical technical applications than the simpler single bubble cavitation. Our research group has recently made an effort to implement these cavitation models in a computer-aided, non-contact optical method for simultaneous flow velocity and pressure field measurements. In this paper, the method will first be presented on a theoretical level, and then evaluated in its practical application for analysis of ultrasonic cavitation on a sonotrode.

\section{Theoretical background}

\subsection{Pressure fluctuations in cavitating flow}

As one of the most important physical quantities in cavitation phenomena, pressure is an inherent part of the Rayleigh-Plesset equation - Eq. (1) [21][22]. Although the RayleighPlesset equation was originally derived for a single spherical bubble, it is also valid for spherically shaped bubble clouds, provided that a correct form of mass and momentum conservation is used (Wang and Brennen [20]). In Eq. (1), the term with the surface tension coefficient is already omitted, as the surface tension was demonstrated to have only a minor effect in various cloud cavitation phenomena, including the acoustic supercavitation on the sonotrode tip (Žnidarčič et al. [23][24]), which will be used in this study to evaluate our optical pressure calculation method.

$$
R\left(1-\frac{\dot{R}}{c}\right) \ddot{R}+\frac{3}{2}\left(1-\frac{\dot{R}}{3 c}\right) \dot{R}^{2}=\frac{1}{\rho_{L}}\left(1+\frac{\dot{R}}{c}+\frac{R}{c} \frac{d}{d t}\right)\left(p-p_{\infty}-4 \frac{\mu_{L}}{R} \dot{R}\right)
$$

In Eq. (1), $R$ represents the equivalent diameter of cavitation cloud, $c$ speed of sound in fluid, $p$ pressure inside the cavitation cloud observed, $\mu_{L}$ fluid kinematic viscosity, $\rho_{L}$ fluid density, and $p_{\infty}$ fluid ambient pressure. According to d'Agostino and Brennen [19], the bubble cloud interfacial velocity $\dot{R}$ is largely subsonic in fluids whose oscillatory dynamics is analyzed in the neighborhood of an equilibrium configuration and at excitation frequencies not much larger that the natural frequencies of individual bubbles. Based on this reasoning it can be

assumed that $\mathrm{c}>>\dot{R}$, therefore a simplified formulation can be derived from Eq. (1) for calculation of pressure fluctuation in cavitation cloud observed [19]:

$$
R \ddot{R}+\frac{3}{2} \dot{R}^{2}=\frac{1}{\rho_{L}}\left(p-p_{\infty}-4 \frac{\mu_{L}}{R} \dot{R}\right)
$$

Taking grayscale image sequence as input data, the equivalent diameter of cavitation cloud $R$ can be assumed to be proportional to the mean image gray level $A$ ( $0 \leq A \leq 1, A=0$ corresponds to black color and $A=1$ to white color) in the monitoring window, $R \propto A$ [11], with proportionality constant $K=R / A$ (unit: $\mathrm{m}$ ). $K$ can be obtained with calibration to an area with known pressure (ambient pressure far away or pressure measured by a reference 
method). The value of $A$ at particular time $t$ can be calculated using Eq. (3), which performs the averaging of $A$ in the spatial and temporal domain.

$$
A(t)=\sum_{i=1}^{i=N} \sum_{j=1}^{M} G(i, j, t) ; \quad G(i, j, t) \epsilon[0 \ldots 1]
$$

The equivalent cloud diameter (i.e. the intensity of gray level fluctuations) was monitored in a rectangular monitoring window of height $M$ and width $N$ (in pixels), where $G$ was the gray level value of a particular pixel at position $(i, j, t)$. The first and second temporal derivatives of $A(\dot{A}$ and $\ddot{A}$, respectively) are calculated using standard numerical derivation schemes. Finally, the instantaneous absolute flow pressure $p(t)$ can be expressed (from Eq. (2) and by considering the proportionality $A=R / K$ ) as:

$$
p(t)=p_{\infty}+K^{2} \rho_{L}\left(A \ddot{A}+\frac{3}{2} \dot{A}^{2}\right)+4 \frac{\mu_{L}}{A} \dot{A}
$$

Resulting time series can be, depending on selection of calculation parameters and software implementation, 1D (integral values of instantaneous pressure within selected window) or 2D (instantaneous pressure fields within selected window).

The presented optical method for pressure calculation is implemented in our flow visualization software ADMflow (available at https://amdflow.net), along with the velocity measurement method which will be presented in the following section. This software was used for all optical calculations of pressure and velocity in this paper. Pressure calculations will be evaluated by comparison to hydrophone-measured pressures.

\subsection{Cavitation structure velocity}

The image sequence previously used for pressure calculation can also be used to compute velocity fields of cavitation structures during the growth and collapse phase. This way, more complete information about the observed flow field can be obtained. Velocity field calculations in this manuscript will be performed according to the algorithm developed by Bajcar et al. [15] and Bizjan et al. [16][17], and implemented in our flow visualization software ADMflow. This velocity calculation algorithm is based on a simplified advectiondiffusion equation for incompressible fluid, formulated as:

$$
\frac{\partial N}{\partial t}+\frac{\partial\left(N v_{x}\right)}{\partial x}+\frac{\partial\left(N v_{y}\right)}{\partial y}=D\left(\frac{\partial^{2} N}{\partial x^{2}}+\frac{\partial^{2} N}{\partial y^{2}}\right)
$$

where the concentration of cavitation phase $N$ is assumed to be proportional to the intensity of gray level $A$ in the monitoring window [16][17]. $D$ represents the diffusion coefficient of the liquid phase, while $v_{\mathrm{x}}$ and $v_{\mathrm{y}}$ are unknown components of velocity vector $\mathbf{v}$ $=\left(v_{x}, v_{y}\right)$ obtained on location $(i, j)$ for each image. In order to close the equation system the temporal and spatial smoothing models were used combined with the image downsampling. Basically, the temporal smoothing penalizes rapid changes in velocity fields with time, having 
an effect of a moving average filter over the unfiltered 2D time series of velocity fields. The spatial smoothing on the other hand penalizes high velocity gradients in the calculation domain, working as a low-pass filter and at the same time allowing a reduction in the number of unknowns in the system of equations. The final solution of the equation system is obtained using the least squares method. Details about the velocity calculation algorithm can be found in references [16] and [17].

\section{Experimental set-up}

Experimental measurements of ultrasonically introduced cavitation below the sonicator probe (also known as the ultrasonic horn) were conducted in a transparent PMMA (poly methyl methacrylate) glass chamber with dimensions of $100 \times 100 \times 100 \mathrm{~mm}$ (experimental setup shown in Fig. 1). The chamber was filled with $600 \mathrm{~mL}$ of distilled water at the ambient temperature $\left(20^{\circ} \mathrm{C}\right)$. Cavitation was generated with an ultrasonic probe of the sonicator (Cole Parmer 4710 series ultrasound homogenizer, model CO300), which had a $3 \mathrm{~mm}$ diameter on its bottom. The ultrasonic probe resonated at a frequency of $20 \mathrm{kHz}$ and was operated at different dimensionless power levels $P$. $P$ was defined as the ratio between the current and maximum allowable sonotrode tip oscillation amplitude $(212 \mu \mathrm{m})$, and was varied between $20 \%$ and $40 \%$. The tip of the probe was placed in the center of the chamber, $5 \mathrm{~mm}$ above the measuring bore and $7 \mathrm{~mm}$ above the hydrophone tip, perpendicular to the chamber bottom and submerged in distillated water, as shown in Fig. 1. This was the smallest possible distance to assure an unobstructed field of view, sufficiently reduce the reverberation effect and at the same time prevent cavitation damage to the hydrophone. The exact placement was achieved using a positioning table which allowed accurate adjustments of sonicator height.

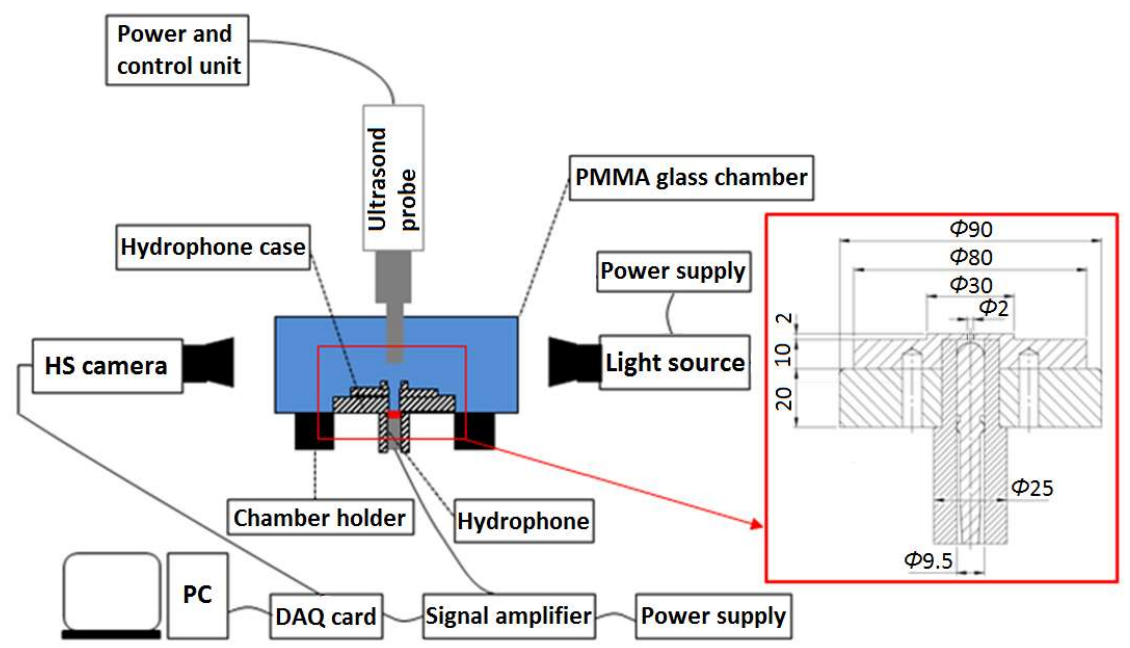

Fig. 1. Experimental set-up

Cavitation induced pressure fluctuations were measured by a piezoelectric hydrophone (Teledyne-Reson TC4013), which had a sensitivity of $-213 \mathrm{~dB} \pm 3 \mathrm{~dB}$ for $1 \mathrm{~V} / \mu \mathrm{Pa}$ output in the 
frequency range of $6-140 \mathrm{kHz}$. Hydrophone output signal was sampled with $200 \mathrm{kHz}$ and acquired to a computer. The hydrophone was mounted in a specially designed casing at the bottom of the glass chamber (detailed view in Fig. 1), so that its tip was located in a bore $2 \mathrm{~mm}$ below the casing bottom.

Simultaneously with the hydrophone measurements, cavitation structures were also visualized by a high-speed camera (Fastec Imaging Hispec 4 Mono) with 85 mm Samyang AE macro lens placed $420 \mathrm{~mm}$ away from the chamber wall. A continuous light source (LED diodes) for illumination of the viewing area was placed on the side of the chamber opposite to the camera so that the ultrasonic probe was placed in the center between them. Highspeed imaging data was acquired with 64031 frames per second and $2 \mu$ s exposure time. Image resolution was $128 \times 80$ pixels, with $0.03 \mathrm{~mm}$ pixel size. The depth of sharpness (approximately $10 \mathrm{~mm}$ ) was sufficient to capture all relevant flow structures.

After acquisition, high-speed images of cavitation structures were imported in the ADMflow software, where velocity and pressure fields were calculated following the methodology presented in the "Theoretical background" section.

\section{Results and discussions}

\subsection{Hydrophone measurements}

Pressure fluctuations measured by the hydrophone were acquired in form of discrete time series of the relative pressure $p_{r}(t)=p(t)-p_{\infty}$ at different sonicator power levels and at an ambient pressure of $p_{\infty}=100 \mathrm{kPa}$. In fig. 2, segments of time series of $p_{r}$ are shown for $20 \%, 30 \%$ and $40 \%$ sonicator power levels, which will later be used for evaluation of our pressure measurement method. 

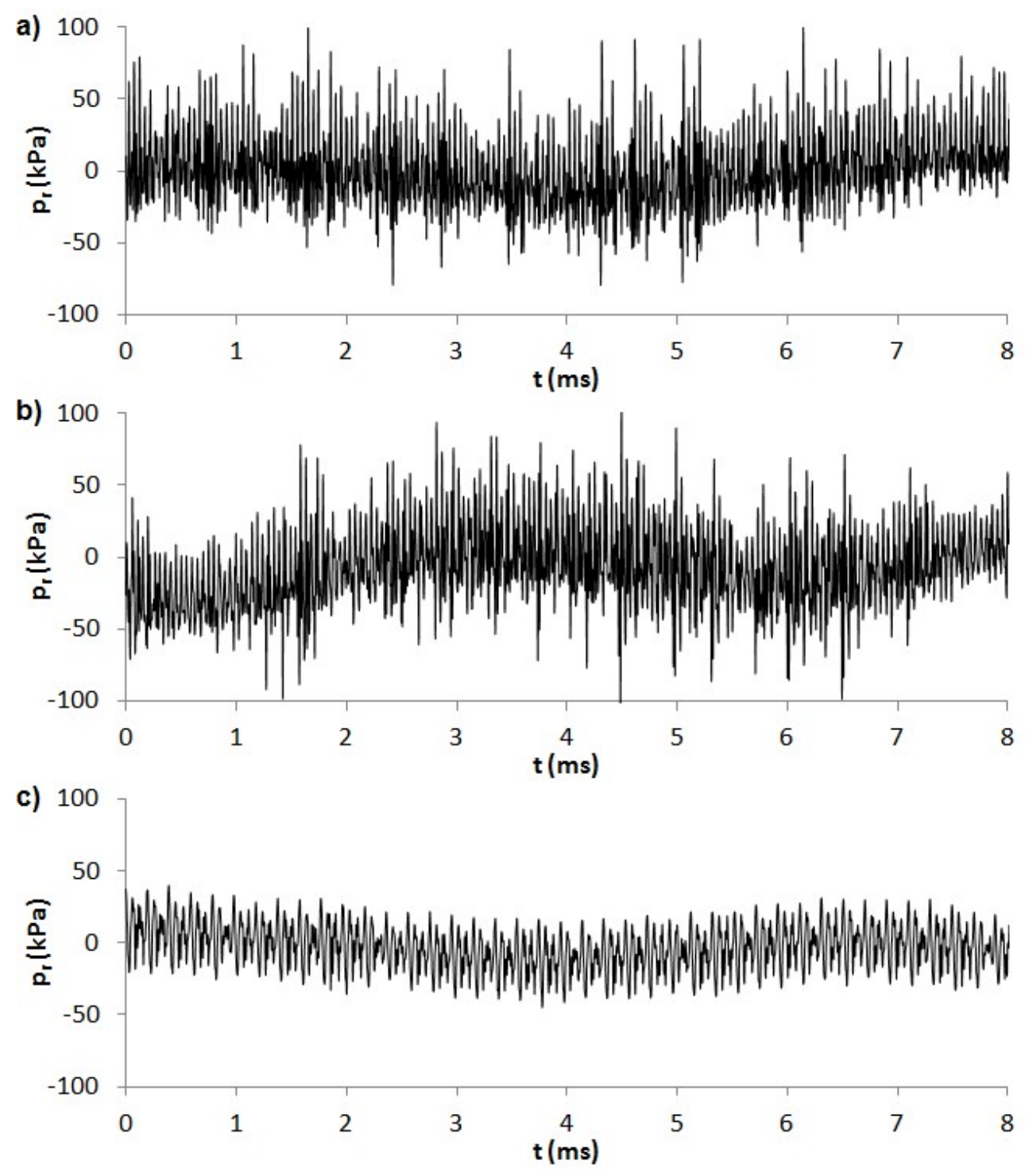

Fig. 2. Time series of pressure fluctuations measured by the hydrophone at different sonicator power levels: $20 \%$ (a), $30 \%$ (b) and $40 \%$ (c)

In Fig. 2, both low and high frequency pressure oscillations can be identified, and the oscillation dynamics at $P=40 \%$ is notably different from those at $P=20 \%$ and $30 \%$, namely due to very different oscillation amplitudes. To characterize the magnitude of pressure fluctuations (and consequently, acoustic cavitation), root-mean-square (RMS) values of time series were calculated. In Fig. 3, pressure RMS values $\left(p_{r, \mathrm{RMS}}\right)$ are shown with respect to the sonicator power level $P$. It can be seen that, until $P=35 \%$, higher sonicator powers lead to an almost linear increase in pressure RMS values, which indicates a more intense cavitation. However, at $\mathrm{P}>36 \%$, the $p_{r, \mathrm{RMS}}$ amplitudes drop sharply, suggesting a transition to a different cavitation regime - acoustic supercavitation [23]. Such transition is usually marked by a reduction of the oscillation frequency of the cavitation structure, which can partly explain the lower RMS value of the pressure signal. 


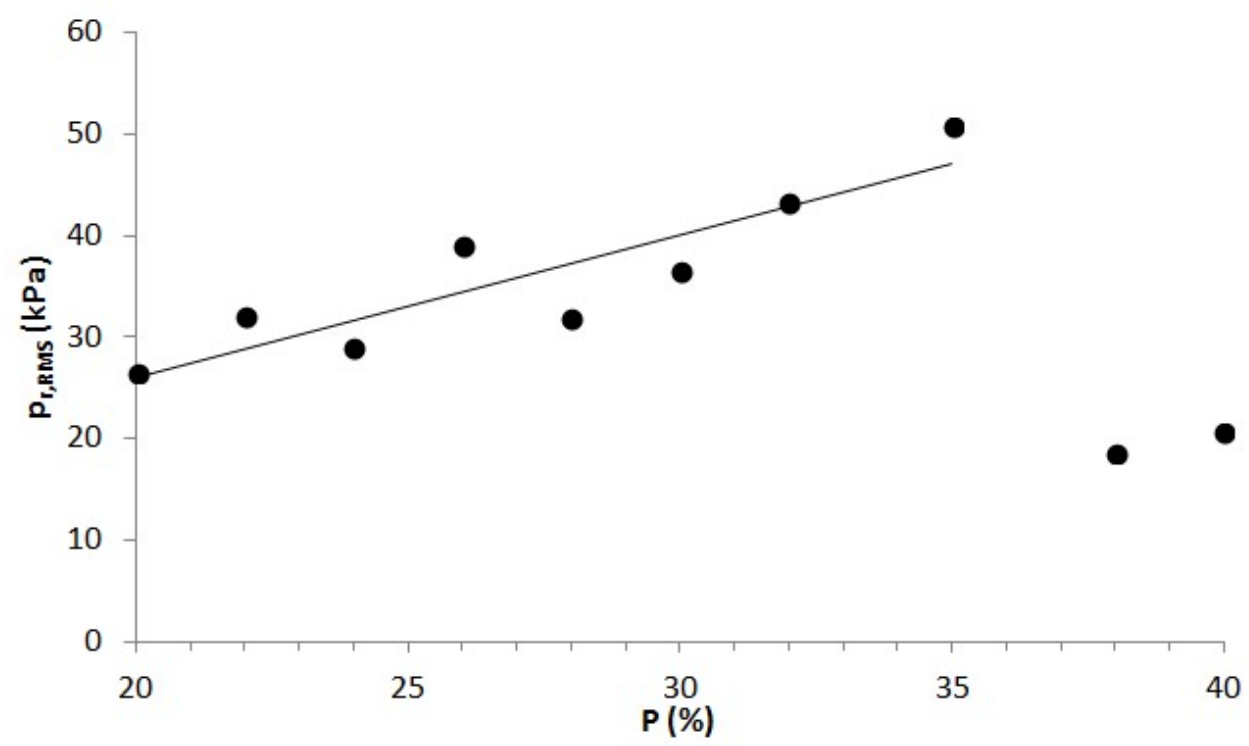

Fig. 1. Influence of the sonicator power level on the RMS value of hydrophone-measured pressure fluctuations

\subsection{Image analysis results}

The region in which the cavitating flow was visualized is presented in Fig. 4, where three consecutive flow images are shown along with the calculation area (window) used for pressure and velocity calculations in ADMflow. In the upper central part of the images, the tip of the ultrasonic probe can be seen. Cavitation at the probe tip occurs in two visually distinct forms: (i) a large coherent vapor structure directly at the tip which frequently appears as a single non-spherical attached bubble, and (ii) much smaller individual bubbles further below, which mostly stream away from the probe tip [23].

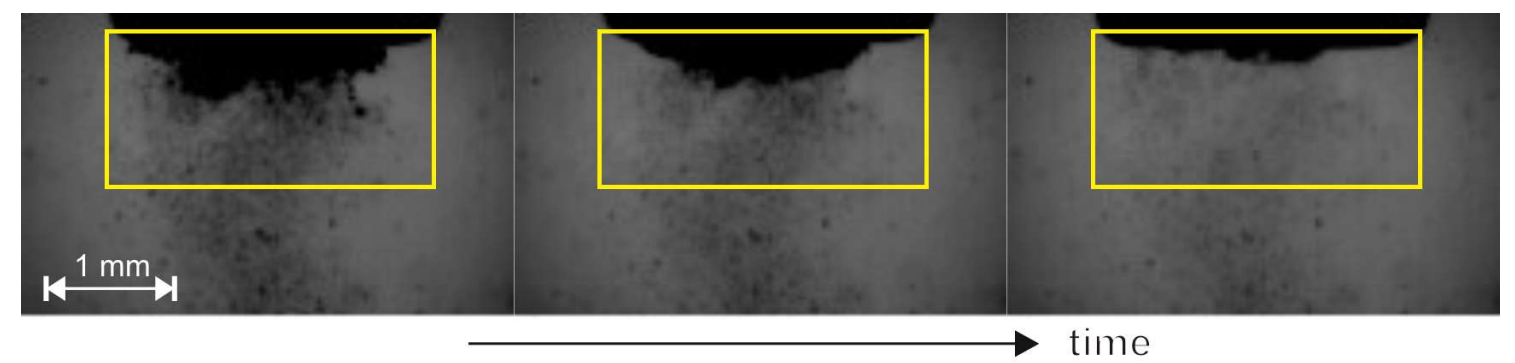

Fig. 2. Consecutive images of cavitation structures (frame separation $15.6 \mu \mathrm{s}$ ) with the pressure and velocity field calculation area (yellow rectangle, gray in printed manuscript)

In Figs. 5, 6 and 7, results of cavitation structure image analysis in ADMflow are presented for different sonicator power levels (20\%, 30\% and $40 \%$, respectively) and compared to the hydrophone data in selected time periods of $100 \mu \mathrm{s}$. In these figures, a) panels show comparison between hydrophone-measured (index HF) and ADMflow-calculated (index ADM) pressure time series, b) panels show velocity directional fields (unit vectors) on 
visualization image background, c) panels calculated fields of dimensionless pressure $\psi$ (Eq. (6)) and d) panels show calculated fields of dimensionless velocity magnitude $\Phi$ (Eq. (7)).

$$
\begin{aligned}
& \Psi=\frac{p-p_{\min }}{p_{\max }-p_{\min }} \\
& \Phi=\frac{v-v_{\min }}{v_{\max }-v_{\min }}
\end{aligned}
$$

Pressure calculation error is defined by Eq. (8), with respect to absolute pressure within the fluid.

$$
e=\frac{\left|p_{\mathrm{r}, \mathrm{HF}}-p_{\mathrm{r}, \mathrm{ADM}}\right|}{p_{\mathrm{r}, \mathrm{HF}}+p_{\infty}}
$$

Pressure and velocity fields in panels b), c) and d) were calculated for three selected frames within presented time series. Calculations were performed according to the presented pressure calculation model (Eqs. (2), (3) and (4)) and the velocity field calculation model (Eq. (5)).

Note that the solid lines which represent pressure time series in Figs. 5a, 6a and 7a were obtained by piecewise polynomial interpolation between measured or resampled data points (i.e. square and diamond shaped markers). 


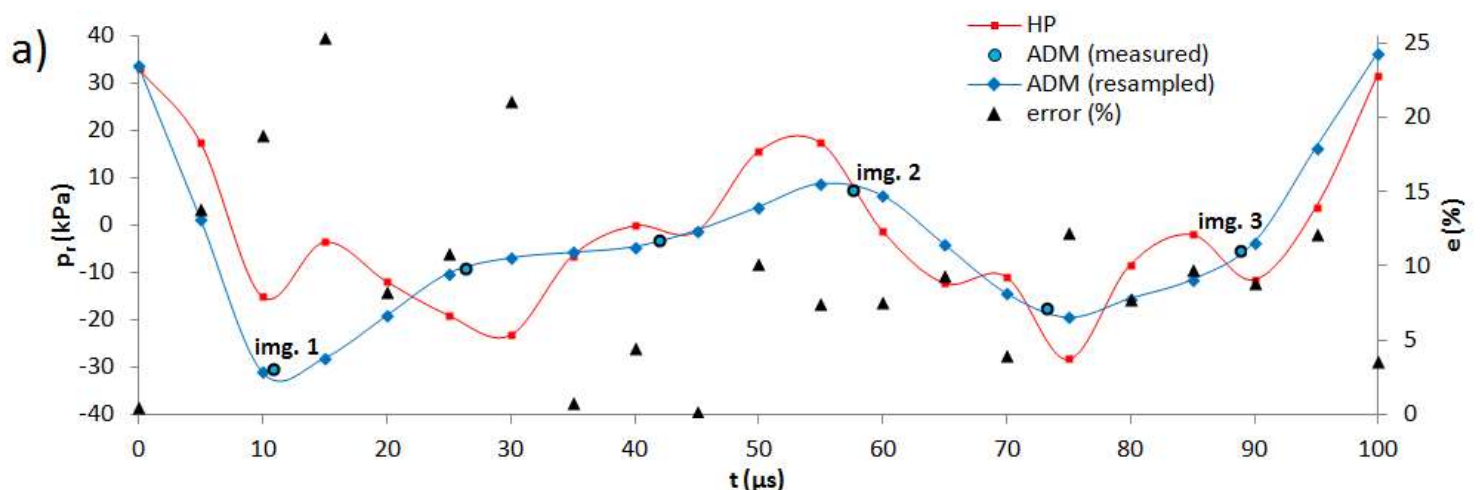

Image 1

b)

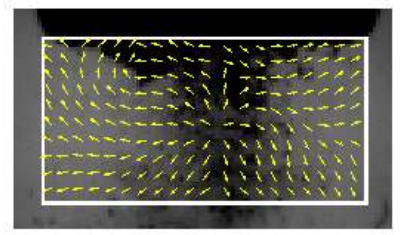

$\mathrm{p}_{\text {min }}=0.4 \mathrm{kPa}, \mathrm{p}_{\text {max }}=156.5 \mathrm{kPa}$
Image 2

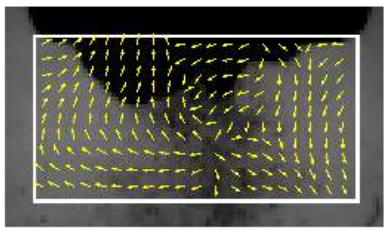

$\mathrm{p}_{\min }=12.0 \mathrm{kPa}, \mathrm{p}_{\max }=178.2 \mathrm{kPa}$

Image 3

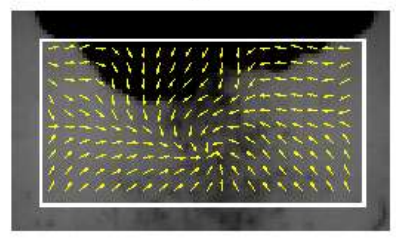

$\mathrm{p}_{\min }=24.7 \mathrm{kPa}, \mathrm{p}_{\max }=149.4 \mathrm{kPa}$

c)
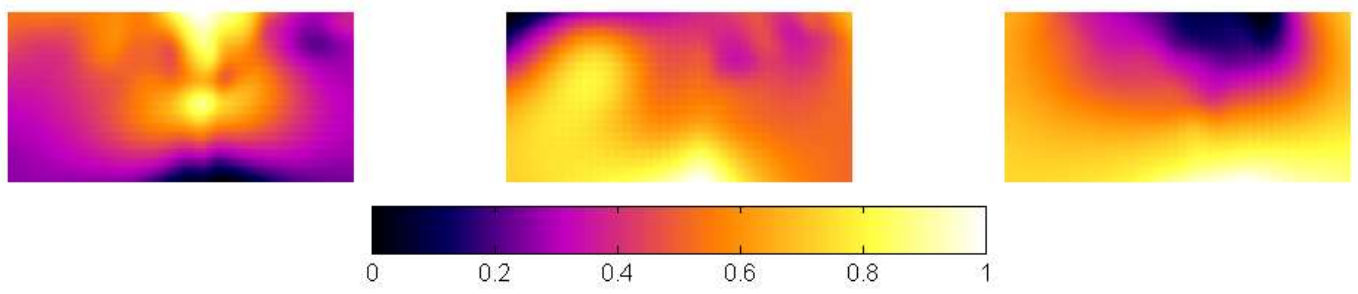

$v_{\min }=0.2 \mathrm{~m} / \mathrm{s}, v_{\max }=21.7 \mathrm{~m} / \mathrm{s}$

$v_{\min }=0.1 \mathrm{~m} / \mathrm{s}, v_{\max }=8.1 \mathrm{~m} / \mathrm{s}$

$v_{\min }=0.3 \mathrm{~m} / \mathrm{s}, v_{\max }=17.1 \mathrm{~m} / \mathrm{s}$

d)
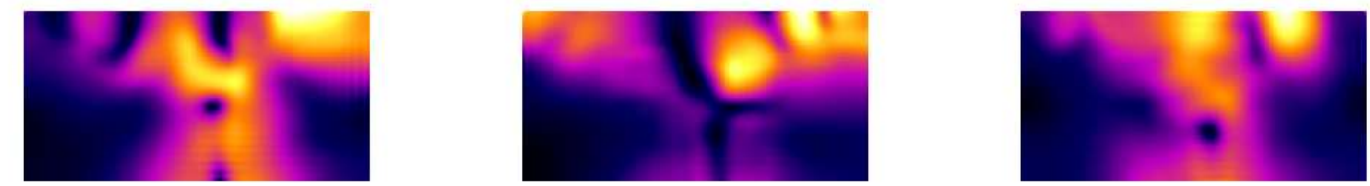

Fig. 3. Pressure and velocity fields at $20 \%$ sonicator power level (mean error $e=9.3 \%$ )

In Fig. 5, cavitation phenomena were investigated at the lowest sonicator power level used (20\%). Selected images in Fig. $5 \mathrm{~b}$ show the cavitation cloud growth and collapse, which is marked by a change of the vapor volume fraction in the region of attached cavitation on the sonicator probe. Process images are overlaid by directional velocity fields, where the flow direction is shown by unit vectors. In each image, the pressure and velocity calculation region is marked by a white rectangle (note that the corresponding fields of dimensionless velocity magnitude $\Phi$ are shown in Fig. $5 \mathrm{~d}$ ). The calculated sequence of dimensionless pressure fields $\psi$ is shown in Fig. 5c. Evident are intense spatial fluctuations of the pressure field topology which take a linear or a spherical shape.

Velocity fields (Fig. $5 b$ and $5 d$ ) characterize the kinematics of the vapor phase in the cavitation cloud and its neighborhood. It is evident that the velocity magnitude increases when the cavitation cloud shrinks (image 1), especially at its edges. Similarly to the pressure distribution fields in Fig. 5c, the regions with intensified kinematics exist in velocity fields. Visual comparison of flow fields in analyzed windows indicates causality between velocity and pressure fields, i.e. the inhomogeneity of pressure distribution can be correlated to 
velocity field distributions around it. Regions of high pressure coincide quite well with the regions of low velocity (and vice versa), which suggests that the continuity and momentum equations are valid for obtained pressure and velocity fields. It can also be seen that velocity vectors are oriented in the direction of maximum pressure gradients. Also, the locations of maximum pressure gradients coincide well with the areas of maximum velocity magnitude.

Besides the two-dimensional flow field analysis, the time series (Fig. 5a) of mean pressures within the calculation window were also analyzed and compared to the hydrophone signal. The blue curve in the diagram in Fig. 5a shows calculated pressure values in the window of interest (resampled to the hydrophone frequency) while the red curve shows pressures measured simultaneously by a hydrophone. In pressure time series diagrams, a significant pressure increase can be observed between image 1 and image 2, which is a consequence of local cavitation structure collapses. The collapse begins as the pressure curve rises, which is seen as formation of a distinct anomaly seen as rapid spatial variation of magnitude in the pressure field and velocity field gradients perpendicular to the pressure extreme. The process is followed by local pressure surges in the region of collapse which have either a linear or a spherical shape. Considering the pressure time series, pressure field distribution and velocity field in the window of interest it can be concluded that the analyzed phenomenon belongs to the local collapse of the cavitation cloud. The optically (ADMflow) calculated pressure time series follows the hydrophone-measured pressure reasonably well, with a mean absolute pressure error of $9.3 \%$.

Similarly to Fig. 5, visualization images presented in Figs. 6 and 7 were selected so that different development phases of cavitation structures were captured. Again, it is evident that the onset of pressure anomalies and the increase in velocity magnitude correspond to a reduction of the vapor phase volume fraction on an integral level, and consequently of the attached cavitation on the sonicator. Local reduction of the attached cavitation (Figs. 6 and 7) is followed by the pressure and velocity field inhomogeneity. The increase of pressure gradients is observed in both analyzed cases at images 2 and 3. Distinct local minima with velocity vectors oriented in the maximum gradient direction are evident in the first phase of the collapse shown in image 1 for both analyzed cases. This indicates movement of the continuum in the direction of the local pressure minimum. This phase is followed by a rapid increase of pressure until reaching the local maximum ( $t=40 \mu \mathrm{s}$ and $t=83 \mu \mathrm{s}$ for Figs. 6 and 7, respectively). Similarly to analysis results presented in Fig. 5, in Figs. 6 and 7 there is mostly a reasonably good agreement between hydrophone-measured and ADMflowcalculated pressure time series, resulting in a 9.3\% mean absolute pressure error for Fig. 6 and $2.5 \%$ error for Fig. 7. Clearly, the hydrophone-measured pressure follows the changes in the magnitude of the pressure field, as well as the magnitude and direction of velocity vectors in the region of local collapses in cavitation clouds. Causality between local pressure anomalies and excited speed oscillations in their close proximity can also be concluded from analyzed cases. 


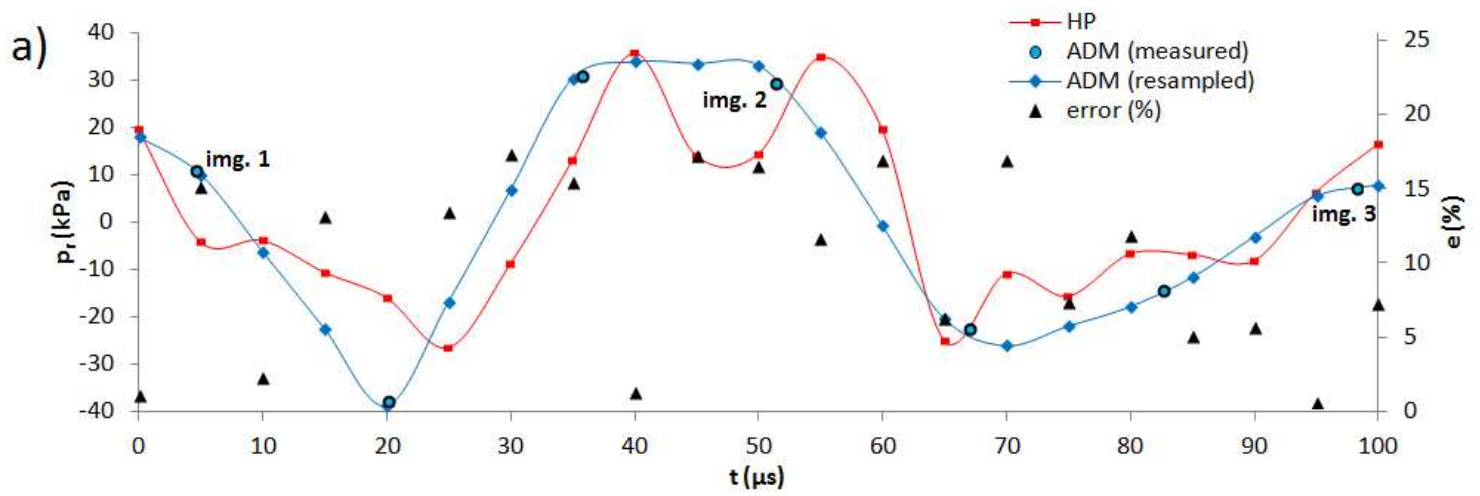

Image 1

Image 2

b)
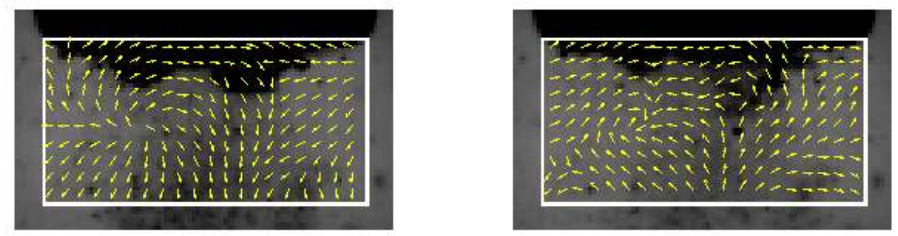

Image 3

$\mathrm{p}_{\min }=39.3 \mathrm{kPa}, \mathrm{p}_{\max }=182.5 \mathrm{kPa} \quad \mathrm{p}_{\min }=38.8 \mathrm{kPa}, \mathrm{p}_{\max }=214.0 \mathrm{kPa} \quad \mathrm{p}_{\min }=2.0 \mathrm{kPa}, \mathrm{p}_{\max }=176.3 \mathrm{kPa}$

c)
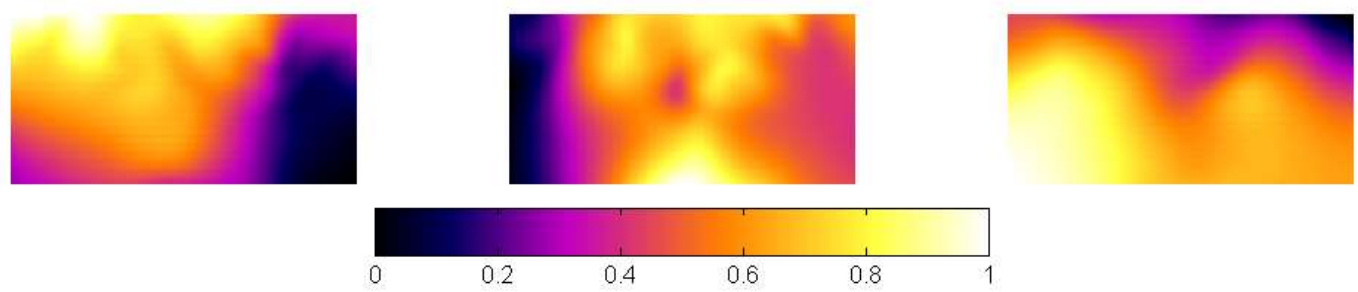

$v_{\min }=0.1 \mathrm{~m} / \mathrm{s}, v_{\max }=21.4 \mathrm{~m} / \mathrm{s}$

$v_{\min }=0.1 \mathrm{~m} / \mathrm{s}, v_{\max }=21.2 \mathrm{~m} / \mathrm{s}$

$v_{\min }=0.1 \mathrm{~m} / \mathrm{s}, v_{\max }=16.8 \mathrm{~m} / \mathrm{s}$

d)
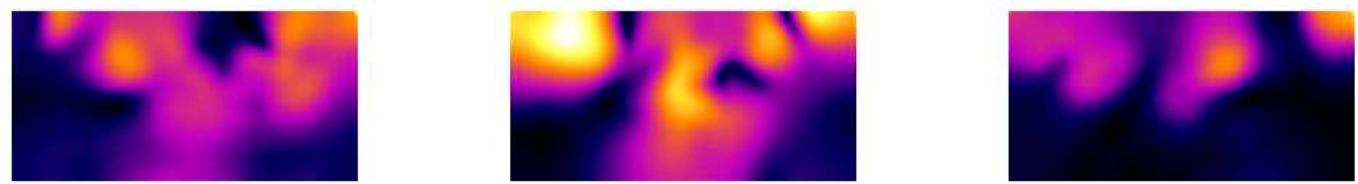

Fig. 4. Pressure and velocity fields at $30 \%$ sonicator power level (mean error $e=10.9 \%$ ) 


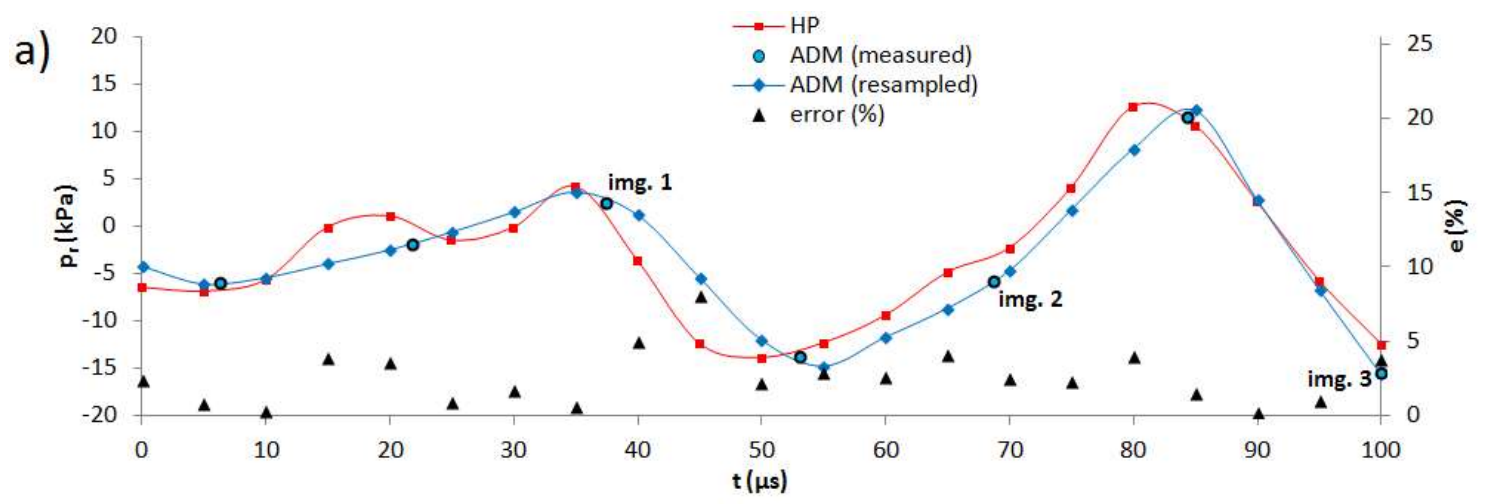

Image 1

b)

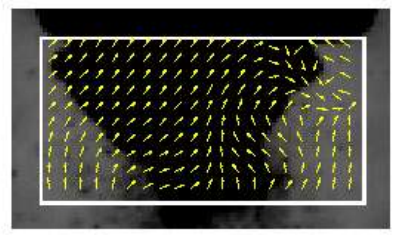

Image 2

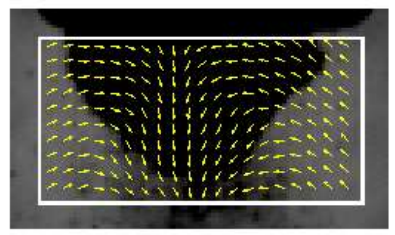

Image 3

$$
\mathrm{p}_{\min }=30.5 \mathrm{kPa}, \mathrm{p}_{\max }=143.4 \mathrm{kPa} \quad \mathrm{p}_{\min }=30.8 \mathrm{kPa}, \mathrm{p}_{\max }=143.5 \mathrm{kPa} \quad \mathrm{p}_{\min }=1.2 \mathrm{kPa}, \mathrm{p}_{\max }=121.6 \mathrm{kPa}
$$

c)
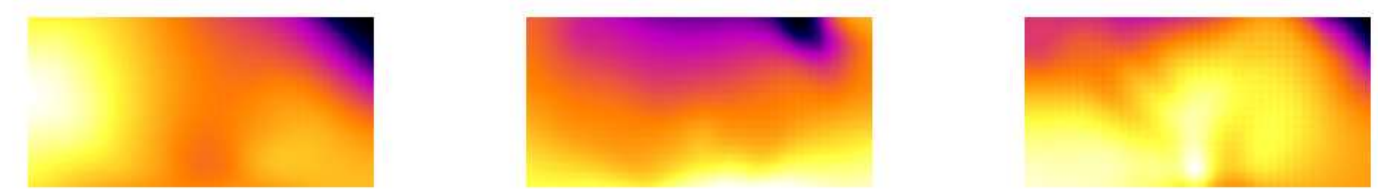

d)

$\Phi$

$v_{\min }=0.0 \mathrm{~m} / \mathrm{s}, v_{\max }=7.0 \mathrm{~m} / \mathrm{s}$
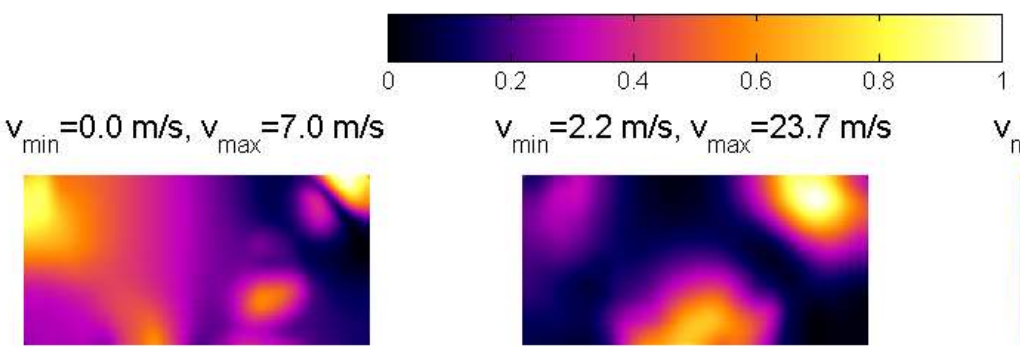

$v_{\min }=2.2 \mathrm{~m} / \mathrm{s}, v_{\max }=23.7 \mathrm{~m} / \mathrm{s}$

$v_{\min }=0.4 \mathrm{~m} / \mathrm{s}, v_{\max }=17.4 \mathrm{~m} / \mathrm{s}$

Fig. 5. Pressure and velocity fields at $40 \%$ sonicator power level (mean error $e=2.5 \%$ )

\subsection{Spectral analysis of pressure time series}

Diagrams in Fig. 8 shows the power spectra of pressure time series obtained by hydrophone measurements (upper panel) and those calculated by our algorithm in ADMflow (lower panel) using flow visualization images as input, at $P=40 \%$. The power spectra comparison will serve for a physical interpretation of flow phenomena in individual ranges of frequency $(f)$, and to assess the suitability of our pressure calculation algorithm as a cavitation diagnostic method. 

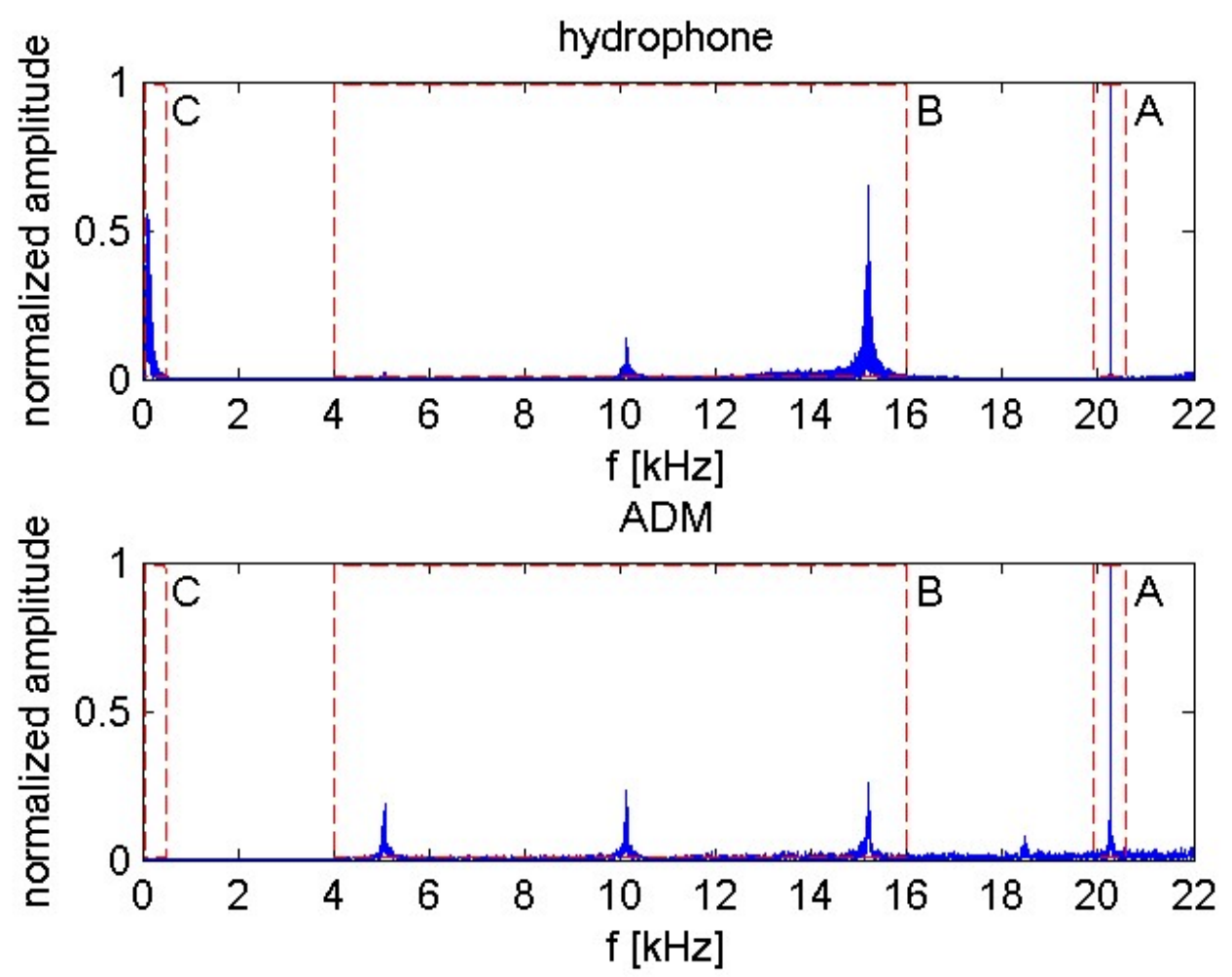

Fig. 8. Pressure fluctuations spectra for $40 \%$ sonicator power level

Power spectra in Fig. 8 can be divided to a low-frequency range $(C)$, medium frequency range $(B)$ and high frequency range $(A)$. In the frequency range $(A)$, it is possible to observe that both methods respond similarly to the excitation frequency of the sonicator $\left(f_{0}=20\right.$ $\mathrm{kHz}$ ), producing a distinct peak with the maximum normalized amplitude in the spectrum. This suggests that periodic oscillations of attached cavitation structures on the sonicator reflect in form of pressure disturbances in the cavitation cloud and result in pulsations at the hydrophone as well. Power spectra in Fig. 8 are only shown for pressures measured at $P=$ $40 \%$, since at $P=20 \%$ and $P=30 \%$, there were no significant peaks in the spectra other than the peak at the sonotrode oscillation frequency. As already pointed out and supported by visualization images (Figs. 5, 6 and 7, panel a), the distinct change in spectral characteristics of the measured pressure signal at $P=40 \%$ are most likely caused by the transition to the acoustic supercavitation regime.

The frequency range (B) $(4-16 \mathrm{kHz})$ is characterized by distinct pressure peaks which appear to be subharmonics of the sonicator excitation frequency $\left(f_{0}=20 \mathrm{kHz}\right)$. According to Žnidarčič et al. [23] [24] and Truong [25], these are likely to be connected to formation of a large single cavity attached to the sonotrode tip, which causes the tip to operate against the vapor phase most of the time, rather than liquid phase. Consequently, the attached large cavity generates its own oscillation frequency (and higher harmonics thereof) by its collapses and rebounds, which can have a very pronounced acoustic emission. In the literature, this frequency is typically reported between $f_{0} / 7$ and $f_{0} / 4$ [23]. Results of our study are consistent with these reports, as we have observed subharmonics of $f_{0}$ at 5,10 and $15 \mathrm{kHz}$, which are the multiples of $f_{0} / 4$. Resulting pressure shocks are also considered to be the main 
mechanism of cavitation erosion effects [8]. When comparing the hydrophone-measured and $A D M$ flow-calculated spectrum, it can be concluded that the positions of frequency peaks in the frequency range $4 \mathrm{~d} 16 \mathrm{kHz}$ is in good agreement for both methods used.

At the lowest frequency range $(C)$, only the hydrophone was able to detect pulsations in the frequency range below $500 \mathrm{~Hz}$ (with most significant peaks occurring between 0 and $150 \mathrm{~Hz}$ ), while the calculated pressure yields zero amplitude in this part of the power spectrum. Considering the fact that the oscillations after the Brennen's model [19] [20] directly reflect the oscillations of the vapor phase, the hydrophone signal pulsations most likely result from the fluid-solid interaction between water and reservoir walls, which is not visible in flow images. Since such low frequency pulsations are not significant for characterization of cavitation phenomena, it can be concluded that our pressure calculation algorithm is able to detect all cavitation-related fluctuations otherwise detectible by the hydrophone, provided that the imaging frame rate is sufficiently high.

To compare power spectra of calculated and hydrophone-measured pressure signals at other sonicator power levels, three-dimensional spectra are shown in Fig. 9. Such representation allows for assessment of sonicator power level effect on cavitation dynamics.

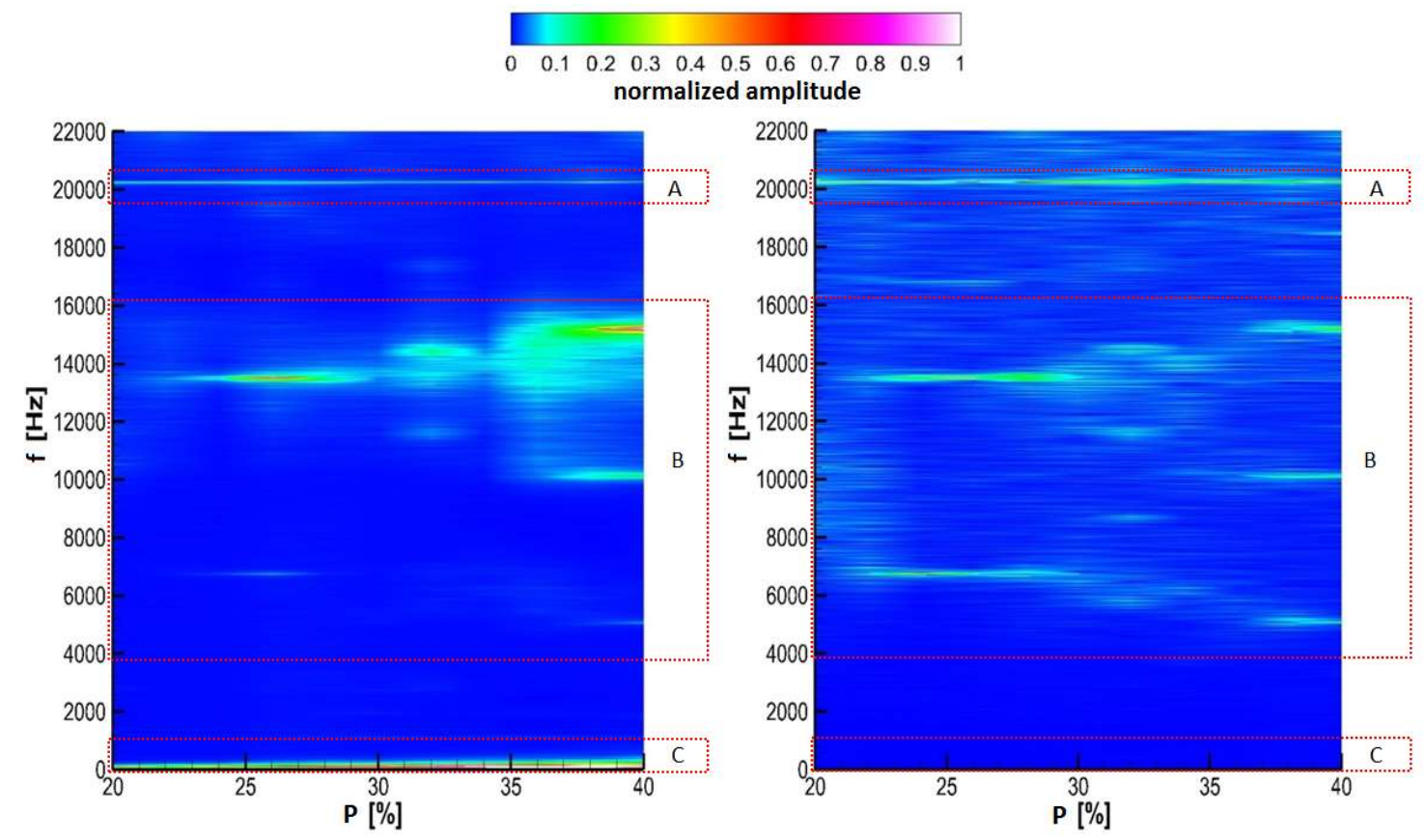

Fig. 9. Frequency specters at different sonicator power levels (left - hydrophone, right ADMflow)

As in Fig. 8, the frequency range can be divided into three frequency subranges ( $A, B$ and $C$ ). The excitation frequency of $20 \mathrm{kHz}$ (subrange $A$ ) is expressed in the entire range of sonicator power levels for modeled and measured pressure signal. Also in the medium frequency range of $4-16 \mathrm{kHz}$ (subrange $B$ ), the spectral amplitude dependence is similar for both measured and calculated pressures. The phenomenon is broad banded and its frequency band expands at higher sonicator power levels as the cavitation collapses become more 
intense. Similarly to Fig. 8, some frequency peaks are more pronounced than the others. Distinct subharmonic peaks of the excitation frequency start to appear above $P=30 \%$. Also, once again, the pressure pulsations occurring at the lowest frequencies are only detectible in the hydrophone pressure signal. As expected, their amplitude and bandwidth increases with the sonicator power level.

\section{Conclusions}

The paper presents a novel non-contact method for simultaneous measurement of pressure and velocity fields of cavitating flows, which uses high-speed imaging data as its input and is implemented in the ADMflow software. The method was tested on the case of attached cavitation below the ultrasonic probe and validated with hydrophone pressure measurements. Experimental results confirm causality between velocity and pressure fields in the phase of cavitation structures collapse, as velocity maxima occur near the maximum pressure gradients. Also, the boundaries of the high velocity areas seem to coincide with the linear or spherical shape of observed cavitation phenomena. Regardless of the sonicator power level and related probe oscillation amplitude, the cavitation cloud collapse causes the pressure and velocity field to become highly inhomogeneous. The progress of local pressure and velocity disturbance is also reflected in local averaged pressure values in the selected regions of interest and has been shown to fit relatively well to the values measured by a hydrophone.

Results of the spectral analysis of pressure signals indicate that the cloud cavitation phenomenon is broad banded and dependent on sonicator excitation power, though cloud oscillations at subharmonic frequencies of the main excitation frequency become more and more pronounced at higher power levels. There is a good agreement in the power spectra for the calculated and hydrophone-measured signal at all except the lowest frequencies. This confirms the suitability of presented visualization method for the diagnosis and prediction of cavitation strokes mechanism, which can be further linked to the effects of cavitation erosion. The presented flow visualization and analysis system can be adapted to different fluid flow examples in a variety of engineering applications.

\section{References}

[1] C.E. Brennen, Cavitation and Bubble Dynamics, Oxford University Press, 1995.

[2] B. Verhaagen, D.F. Rivas, Measuring cavitation and its cleaning effect. Ultrasonics Sonochemistry, 29, 619-628, 2016.

[3] R.B. Wade, A.J. Acosta, Experimental observation on the flow past a plano-convex hydrofoil, Transaction of ASME, Journal of Basic Engineering, 87, 273-283, 1966.

[4] A. Kubota, H. Kato, H. Yamaguchi, M. Maeda, Unsteady structure measurement of cloud cavitation on a foil section using conditional sampling technique, Journal of Fluids Engineering, 111(2), 204-210, 1989.

[5] G.E. Reisman, C.E. Brennen, Pressure pulses generated by cloud cavitation, ASME Fluids Engineering Division Conference, 1996.

[6] J.P. Franc, E. Janson, P. Morel, C. Rebattet, M. Riondet, Visualizations of leading edge cavitation in an inducer at different temperatures. Proceedings of the 4 International Symposium on Cavitation CAV2001, California Institute of Technology, Pasadena, CA USA, 2001. 
[7] K. Kato, H. Dan, Y. Matsudaira, Lock-in Phenomenon of Pitching Hydrofoil with Cavitation Breakdown (Unsteady Fluid Force Characteristics and Visualization of Flow Structures), Department od Systems Engineering Science, Tokyo Metropolitan Institute of Technology, 6-6 Asahigaoka, Hino-shi, Tokyo 1910065, Japan, 2006.

[8] I. Tzanakis, G.S.B. Lebon, D.G. Eskin, K.A. Pericleous, Characterizing the cavitation development and acoustic spectrum in various liquids, Ultrasonics Sonochemistry (2016), doi: http://dx.doi.org/10.1016/j.ultsonch.2016.06.034

[9] D. M. Leppinen, S.B. Dalziel, A light attenuation technique for void fraction measurement of microbubbles, Experiments in Fluids, vol.30, no. 2, 214-220, 2001.

[10] G.E. Reisman, Y.C. Wang, C.E. Brennen, Observations of shock waves in cloud cavitation, Journal of Fluid Mechanics, 355, 255-283, 1998.

[11] A. Osterman, M. Hočevar, B. Širok, M. Dular, Characterization of incipient cavitation in axial valve by hydrophone and visualization. Experimental Thermal and Fluid Science, 33, 620-629, 2009.

[12] Franc, J.P., Michel, J.M. Fundamentals of cavitation. Springer, Dordrecht 2004.

[13] J.L. Barron, D.J. Fleet, S. Beauchemis, Performance of optical flow techniques. Int J Comput Vis, 12, 43-77, 1994.

[14] H. Zimmer, A. Bruhn, W. Joachim, Optic flow in harmony. Int J Comput Vis, 93, 368-88, 2011.

[15] T. Bajcar, B. Širok, M. Eberlinc, Quantification of flow kinematics using computer-aided visualization. Strojniški vestnik - Journal of Mechanical Engineering, 55(4), 215-223, 2009.

[16] B. Bizjan, A. Orbanić, B. Širok, T. Bajcar, L. Novak, B. Kovač Flow Image Velocimetry Method Based on Advection-Diffusion Equation, Strojniški vestnik - Journal of Mechanical Engineering, 60(78), 483-494, 2014. DOI: http://dx.doi.org/10.5545/sv-jme.2013.1614

[17] B Bizjan, A Orbanić, B Širok, B Kovač, T Bajcar, I Kavkler. A computer-aided visualization method for flow analysis. Flow Measurement and Instrumentation, 38, 1-8, 2014.

[18] M.S. Plesset, A. Prosperetti, Bubble Dynamics and Cavitation. Annual Review of Fluid Mechanics, 9, 145-185, 1977.

[19] L. d'Agostino, C. E. Brennen, Linearized dynamics of spherical bubble clouds. J. Fluid Mech. 199, 155-176, 1989.

[20] Y.C. Wang, C.E. Brennen, Numerical Computation of Shock Waves in a Spherical Cloud of Cavitation Bubbles. Journal of Fluids Engineering, 121 (4), 872-880, 1999.

[21] M. Shimada, T. Kobayashi, Dynamics of Cloud cavitation and Cavitation erosion, Proceedings of the 1999 ASME Fluids Engineering Summer meeting 1999, San Francisco, 1-6, 1999.

[22] A. Prosperetti, Bubble phenomena in sound Fields: Part one. Ultrasonics, March, 69-78, 1984.

[23] A. Žnidarčič, R. Mettin, C. Cairós, M. Dular, Attached cavitation at a small diameter ultrasonic horn tip. Physics of Fluids, 26, 023304, 2014.

[24] A. Žnidarčič, R. Mettin, M. Dular, Modeling Cavitation in a Rapidly Changing Pressure Field Application to a Small Ultrasonic Horn. Modeling cavitation in a rapidly changing pressure field Application to a small ultrasonic horn. 22, 482-492, 2015.

[25] V. A. Truong, A study of impulsive pressure distribution of cavitation generated by a high frequency vibrational probe, J. Sci. Technol. (Technical Universities Pub. Hanoi, Vietnam) 73, 78-84, 2009. 